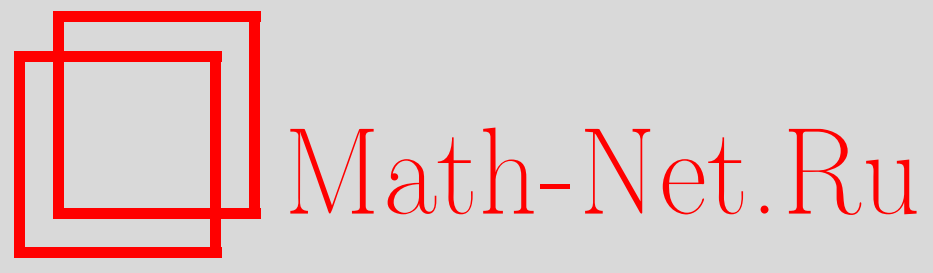

А. В. Гурская, Ширина распада бозона Хиггса на два фотона в однопетлевом приближении с учетом СР-нарушения в HMCCM, Вестн. Сам. гос. техн. ун-та. Сер. Физ.-мат. науки, 2015, номер 4, 650657

DOI: https://doi.org/10.14498/vsgtu1391

Использование Общероссийского математического портала MathNet.Ru подразумевает, что вы прочитали и согласны с пользовательским соглашением

http://www . mathnet.ru/rus/agreement

Параметры загрузки:

IP: 52.205 .19 .152

26 апреля 2023 г., 13:43:00

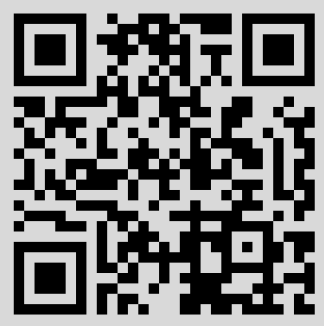


УДК 539.12

\section{ШИРИНА РАСПАДА БОЗОНА ХИГГСА НА ДВА ФОТОНА В ОДНОПЕТЛЕВОМ ПРИБЛИЖЕНИИ С УЧЕТОМ СР-НАРУШЕНИЯ В НМССМ}

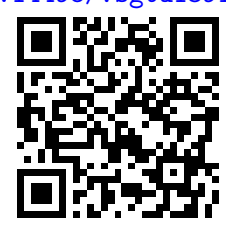

\section{А. В. Гурская}

Самарский государственный университет,

Россия, 443011, Самара, ул. Академика Павлова, 1.

\section{Аннотация}

В статье рассматривается проблема расширения стандартной модели физики элементарных частиц. Основное внимание уделено неминимальной суперсимметричной модели. На сегодняшний день ее рассмотрение является наиболее приемлемым по сравнению с другими разрабатываемыми суперсимметричными теориями. Мы также рассматриваем нарушение СР-инвариантности в секторе Хиггса. При нахождении физических состояний бозонов Хиггса мы находим локальный минимум потенциала Хиггса. Эту процедуру необходимо выполнить для того, чтобы получить стабильное состояние вакуума. Далее произведены расчеты ширины распада бозона Хиггса на два фотона. Мы используем метод квантово-полевой теории возмущений совместно с фейнмановским диаграммным подходом. Результаты расчётов представлены графически. Показано влияние СР-нарушающей фазы на величину ширины распада. Также приведены зависимости масс бозонов Хиггса от фазы СРнарушения в древесном приближении. Некоторые параметры модели не определены на эксперименте, а заключены в некотором диапазоне значений, что дает возможность рассматривать различные сценарии нескольких бозонов Хиггса. В частности, в случае СР-нарушения возможно существование бозона Хиггса с массой меньше 125 ГэВ. Это объясняется отсутствием определенного СР-состояния этой частицы.

Ключевые слова: LHC, бозон Хиггса, нарушение CP-инвариантности, суперсимметрия, ширина распада, однопетлевые вклады.

doi: http://dx.doi.org/10.14498/vsgtu1391

Введение. Бозон Хиггса с массой 125 ГэВ наблюдается в экспериментах на LHC в основных процессах распада: $H \rightarrow \gamma \gamma$ на два фотона, на пару промежуточных $Z$ - и $W$-бозонов с последующим распадом на четыре лептона $H \rightarrow Z Z^{*} / W W^{*} \rightarrow 4 l[1,2]$. Первый канал является не столь вероятным для данного значения массы, но наиболее «чистым» для наблюдения, так как в этом случае фоновые процессы значительно ослаблены. Вероятность

(C) 2015 Самарский государственный технический университет.

\section{Образец для цитирования}

Гурск а я А. В. Ширина распада бозона Хиггса на два фотона в однопетлевом приближении с учетом СР-нарушения в НМССМ // Вестн. Сам. гос. техн. ун-та. Сер. Физ.-мат. науки, 2015. Т. 19, № 4. C. 650-657. doi: 10.14498/vsgtu1391.

Сведения об авторе

Альбина Валентиновна Гурская (a-gurska@yandex.ru), аспирант, каф. общей и теоретической физики. 
наблюдения других двух каналов значительно выше. Далее в статье мы будем рассматривать только распад на два фотона.

Необходимо отметить также, что эксперименты на LHC посвящены в целом не только изучению свойств бозона Хиггса, но и поиску так называемой «новой физики», то есть эффектов, выходящих за рамки Стандартной модели (CM). Расширение СМ становится неизбежным в процессе решения ее внутренних трудностей касательно масс нейтрино, барионной асимметрии, темной материи и др. Несмотря на то, что наблюдаемый бозон Хиггса по своим физическим свойствам пока что удовлетворяет СМ, ситуация может оказаться не такой простой в действительности. В статье Д. И. Казакова [3], а также в ранней работе авторов [4] рассматриваются возможности реализации «нестандартного» бозона Хиггса в экспериментах на LHC. Во-первых, бозон Хиггса вполне может оказаться связанным состоянием, то есть, тогда должны существовать другие более тяжелые возбужденные состояния, помимо основного наблюдаемого. Во-вторых, бозонов Хиггса может быть изначально несколько, причем с разными свойствами, и вполне может оказаться, что мы наблюдаем только один из множества бозонов Хиггса. Последнее возможно при расширении именно сектора Хиггса.

Расширенный сектор Хиггса также может дать теории новые источники нарушения СР-инвариантности. Это нарушение, согласно А. Д. Сахарову [5], является одной из причин барионной асимметрии Вселенной. Подробно способы введения СР-нарушения в сектор Хиггса рассмотрены в работе [6]. Учет нарушения данной симметрии может дать более богатую картину физических свойств бозонов Хиггса. Например, может существовать легкий бозон Хиггса с массой меньше 125 ГэВ, который ненаблюдаем на эксперименте в данный момент именно за счет того, что не обладает определенной СР-четностью.

Интересны также суперсимметричные расширения СМ. Идея суперсимметрии заключается во взаимном преобразовании бозонов и фермионов. В рамках СМ суперсимметрия нереализуема из-за недостаточного соответствия степеней свободы бозонов и фермионов, поэтому простейшей суперсимметричной моделью может быть минимальное расширение СМ, при котором каждой частице в соответствие ставится частица-суперпартнер (счастица). Отметим также, что такая модель уже должна содержать два дублета скалярных полей Хиггса, исходя из требований киральности полей в лагранжиане. Таким образом, мы получаем минимальную суперсимметричную стандартную модель (MCСM). Она долгое время была популярна, но в ней оставалась нерешенной так называемая $\mu$-проблема, которая нашла успешное решение при введении в теорию дополнительного синглетного суперполя в сектор Хиггса. Полученная модель стала называться неминимальной суперсимметричной стандартной моделью (НMCСМ). В ней содержится семь физических состояний бозонов Хиггса. На сегодняшний день рассмотрение этой модели является наиболее приемлемым.

Возвращаясь к проблеме СР-нарушения, скажем еще, что возможность напрямую исследовать ее в распадах бозона Хиггса на LHC пока нереализуема. Изучение CP-симметрии проводится на детекторе LHCb, который «не видит» бозон Хиггса. Однако нас интересует, какое влияние может оказывать нарушение СР-инвариантности в секторе Хиггса на одну из важнейших характеристик процессов распадов - их ширину. Таким образом, целью дан- 
ной работы является исследование влияние СР-нарушения на двухфотонный распад бозона Хиггса в НМССМ.

1. Нарушение СР-инвариантности в секторе Хиггса НМССМ. Нарушение СР-инвариантности в секторе Хиггса было рассмотрено ранее в двухдублетной модели [7] и в МССМ [8,9]. Авторы использовали метод эффективного потенциала для нахождения масс физических состояний бозона Хиггса через локальный минимум потенциала. Нарушение СР-инвариантности связано с присутствием в потенциале Хиггса комплексных параметров. Из-за этого процедура нахождения локального минимума усложняется и, как было показано авторами, не всегда корректна в случае только лишь спонтанного нарушения СР-инвариантности. Авторы пришли к выводу, что необходимо также присутствие членов, явно нарушающих СР-симметрию.

Основываясь на этих выводах, мы рассматриваем только явное CР-нарушение. Наиболее естественно его можно ввести через комплексные юкавские константы. Таким образом, мы можем рассматривать изначально теорию с СР-сохранением, как в работе [4], получив физические состояния бозонов Хиггса, а затем смешав СР-четные состояния с СР-нечетными дополнительным поворотом по аналогии с работой [8].

2. Ширина распада бозона Хиггса на два фотона в однопетлевом приближении. Процесс $H \rightarrow \gamma \gamma$ является принципиально петлевым. Исходя из основных положений теории возмущений с использованием диаграммной техники Фейнмана, однопетлевые вклады в данный процесс можно расписать так, как показано на рис. 1.

Мы можем рассматривать в качестве лидирующих вкладов диаграмму с фермионами 1, суперсимметричные вклады чарджино 2 и сфермионов 5, 16, а также имеет смысл рассмотреть вклады от $W$-бозона ввиду массивности данных частиц по сравнению с остальными.

Формула расчета ширины распада бозона Хиггса на два фотона в общем виде выглядит следующим образом:

$$
\Gamma\left(H_{i} \rightarrow \gamma \gamma\right)=\frac{M_{H_{i}}^{3} \alpha}{256 \pi^{3} v^{2}}\left[\left|S_{i}^{\gamma}\left(M_{H_{i}}\right)\right|^{2}+\left|P_{i}^{\gamma}\left(M_{H_{i}}\right)\right|^{2}\right],
$$

где

$$
\begin{gathered}
S_{i}^{\gamma}\left(M_{H_{i}}\right)=2 \sum_{f=b, t, \tilde{\chi}_{1}^{ \pm}, \tilde{\chi}_{2}^{ \pm}} N_{C} Q_{f}^{2} g_{H_{i \bar{f} f}}^{S} \frac{v}{m_{f}} F_{s f}\left(\tau_{i f}\right)- \\
-\sum_{\tilde{f}_{j}=\tilde{t}_{1}, \tilde{t}_{2}, \tilde{b}_{1}, \tilde{b}_{2}, \tilde{\tau}_{1}, \tilde{\tau}_{2}} N_{C} Q_{f}^{2} g_{H_{i} \tilde{f}_{j}^{*} f_{j}} \frac{v^{2}}{2 m_{\tilde{f}_{j}}} F_{0}\left(\tau_{i \tilde{f}_{j}}\right)- \\
-g_{H_{i} V V} F_{1}\left(\tau_{i W}\right)-g_{H_{i} H^{+}} H^{-} \frac{v^{2}}{2 M_{H^{ \pm}}^{2}} F_{0}\left(\tau_{i H^{ \pm}}\right), \\
P_{i}^{\gamma}\left(M_{H_{i}}\right)=2 \sum_{f=b, t, \tilde{\chi}_{1}^{ \pm}, \tilde{\chi}_{2}^{ \pm}} N_{C} Q_{f}^{2} g_{H_{i \bar{f} f}}^{P} \frac{v}{m_{f}} F_{p f}\left(\tau_{i f}\right) .
\end{gathered}
$$

Здесь $\tau_{i x}=M_{H_{i}}^{2} / 4 m_{x}^{2}, N_{C}=3$ для (с)кварков и $N_{C}=1$ для стау и чарджино соответственно. 

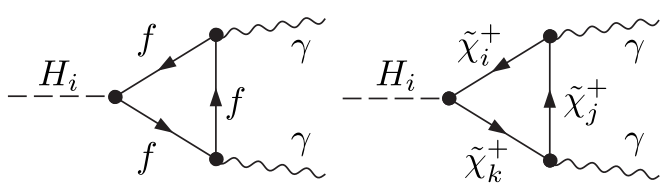

1

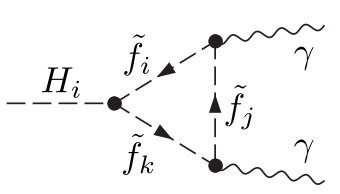

5

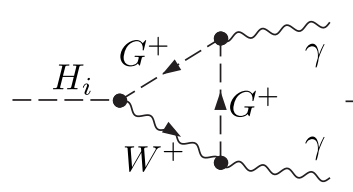

9

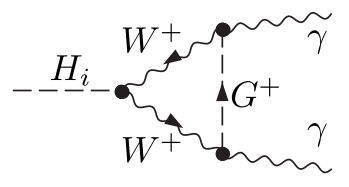

13

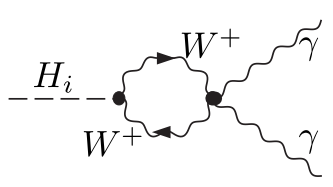

17

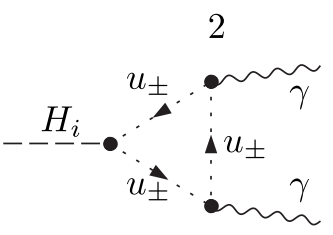

6

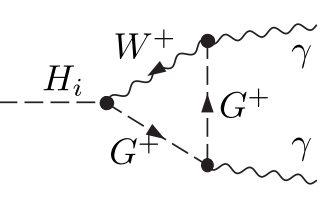

10

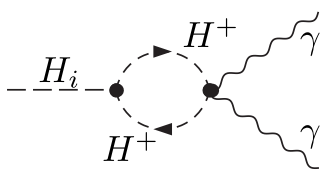

14

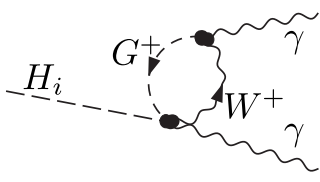

18

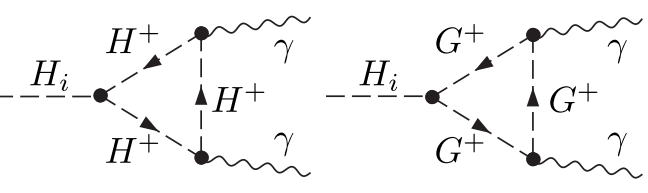

3

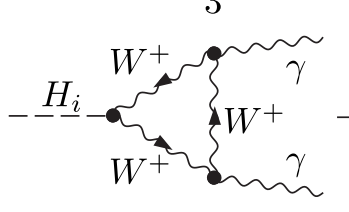

7
4

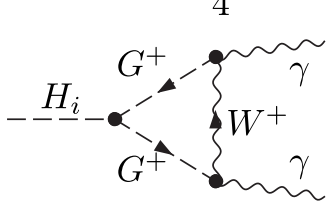

8

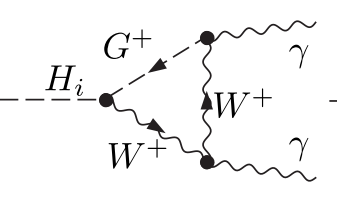

11

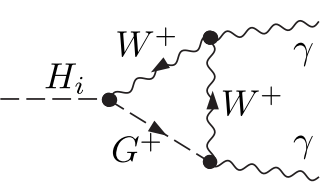

12

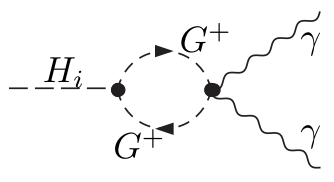

15

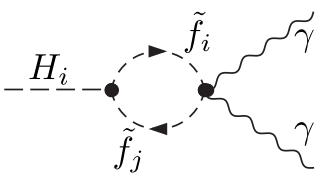

16

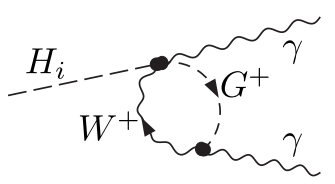

19

Рис. 1. Система диаграмм Фейнмана, определяющих петлевой вклад в процесс распада бозона Хиггса на два фотона в $\operatorname{HMCCM}\left(H_{i}\right.$ - нейтральный бозон Хиггса, $\left.i=1,2, \ldots, 5\right)$

[Figure 1. The system of Feynman diagrams which determine the loop contribution into the

Higgs boson decay to two photons in NMSSM $\left(H_{i}\right.$ is neutral Higgs boson, $\left.i=1,2, \ldots, 5\right)$ ]

Формфакторы $F_{s f}, F_{p f}, F_{0}$ и $F_{1}$ могут быть представлены в терминах масштабной функции $f(\tau)$ :

$$
\begin{gathered}
F_{s f}(\tau)=\tau^{-1}\left[1+\left(1-\tau^{-1}\right) f(\tau)\right], \quad F_{p f}=\tau^{-1} f(\tau), \\
F_{0}=\tau^{-1}\left[-1+\tau^{-1} f(\tau)\right], \quad F_{1}(\tau)=2+3 \tau^{-1}+3 \tau^{-1}\left(2-\tau^{-1} f(\tau)\right) .
\end{gathered}
$$

Функция $f(\tau)$ является интегральной функцией:

$f(\tau)=-\frac{1}{2} \int_{0}^{1} \frac{\mathrm{d} y}{y} \ln [1-4 \tau y(1-y)]=\left\{\begin{array}{cl}\arcsin ^{2}(\sqrt{\tau}): & \tau \leqslant 1 \\ -\frac{1}{4}\left[\ln \left(\frac{\sqrt{\tau}+\sqrt{\tau-1}}{\sqrt{\tau}-\sqrt{\tau-1}}\right)-i \pi\right]^{2}: & \tau \geqslant 1 .\end{array}\right.$ 


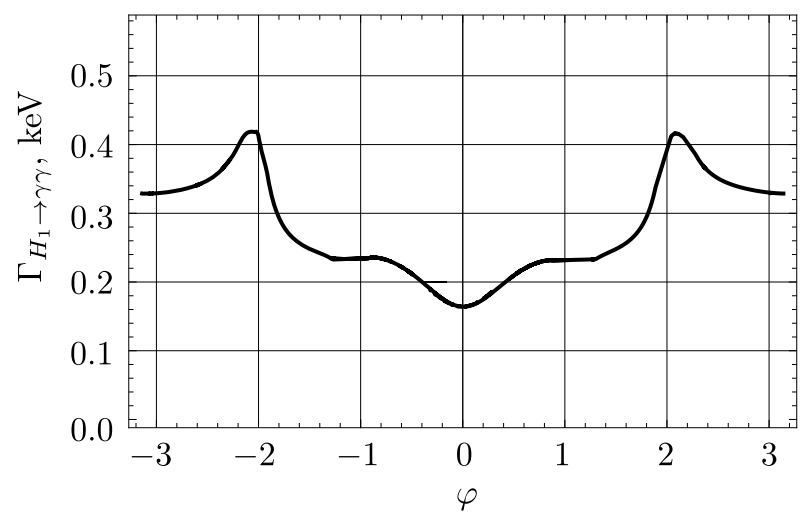

Рис. 2. Зависимость ширины распада бозона Хиггса с массой 125 ГэВ от фазы СР-нарушения $\varphi$ [Figure 2. The dependence of widths of the Higgs boson decay with mass of $125 \mathrm{GeV}$ on the CP violation phase $\varphi$ ]

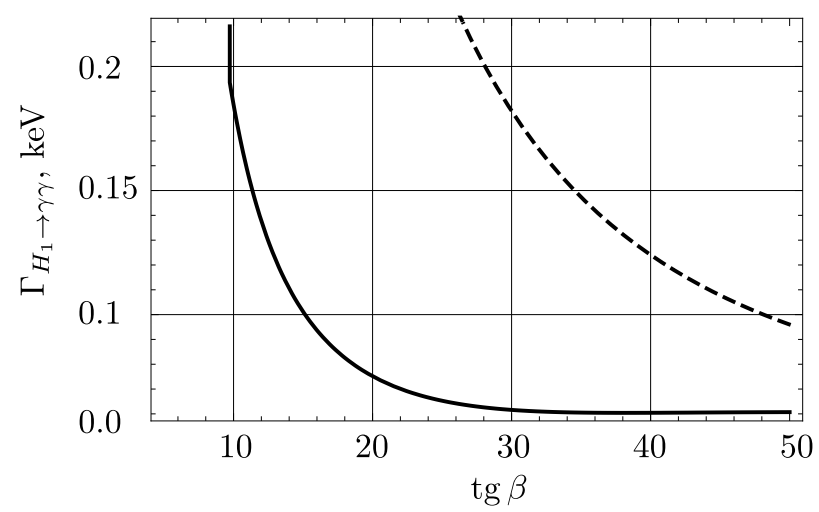

Рис. 3. Зависимость ширины распада бозона Хиггса с массой 125 ГэВ от $\operatorname{tg} \beta$ - тангенса угла смешивания нефизических заряженных и нейтрального СР-нечетного полей Хиггса (сплошная линия - в случае СР-нарушения; пунктир - в случае СР-сохранения) при фиксированной фазе $\varphi=\pi / 2$

[Figure 3. The dependence of widths of the Higgs boson decay with mass of $125 \mathrm{GeV}$ on $\operatorname{tg} \beta-$ the tangent for mixing charged and neutral CP odd Higgs fields (solid line in the case of CP violation; the dotted line in the case of CP conservation) at a fixed phase $\varphi=\pi / 2$ ]
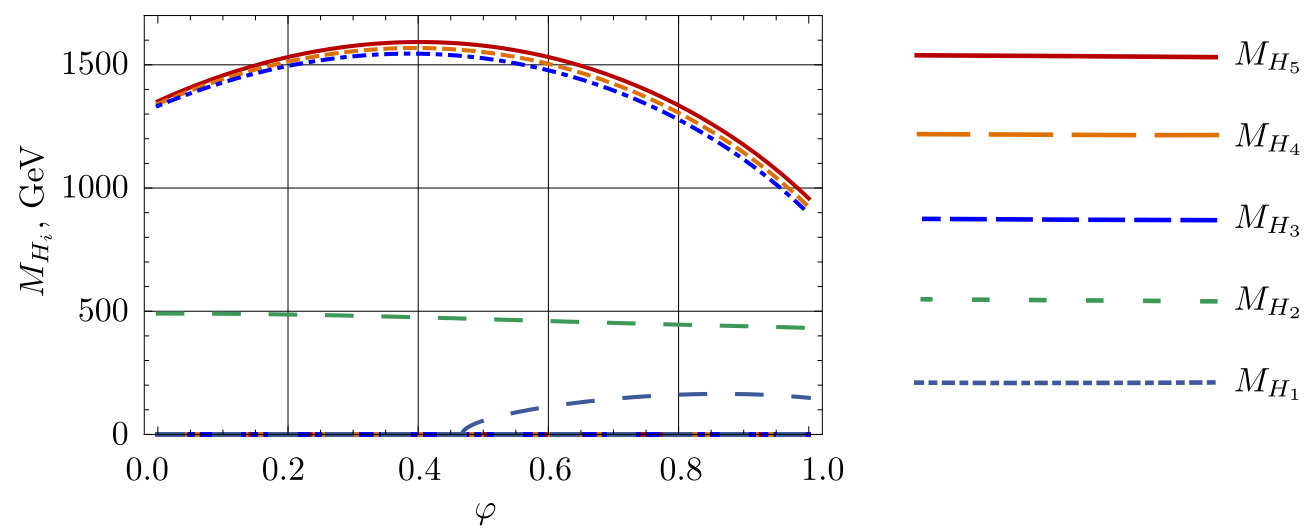

Рис. 4. (online в цвете) Зависимость массы бозона Хиггса от фазы СР-нарушения $\varphi$ [Figure 4. (color online) The dependence of the Higgs boson mass on the phase of CP violation $\varphi$ ] 
3. Численный анализ и результаты. НМССМ содержит ряд свободных параметров, которые не определены на эксперименте, но на сегодняшний день имеют некоторые ограничения:

$$
\begin{gathered}
1.0<\operatorname{tg} \beta \leqslant 60, \quad M_{1}=M_{2}, \quad 100 \text { ГэВ } \leqslant M_{2} \leqslant 2000 \text { ГэВ } \\
0.0001 \leqslant \lambda \leqslant 0.7, \quad 0 \leqslant \kappa \leqslant 0.65 ; \\
0 \leqslant A_{\lambda} \leqslant 1000 \text { ГэВ, }-100 \text { ГэВ } \leqslant A_{\kappa} \leqslant-10 \text { ГэВ. }
\end{gathered}
$$

Таким образом, ширину распада можно рассматривать в данной модели как функцию этих свободных параметров. Дополнительным параметром будет фаза СР-нарушения $\varphi$, которая есть не что иное, как угол смешивания СР-четных и СР-нечетных физических состояний бозона Хиггса.

На рис. 2 представлена зависимость ширины распада бозона Хиггса с массой 125 ГэВ в зависимости от фазы СР-нарушения $\varphi$. На рис. 3 представлены два графика зависимости ширины двухфотонного рапада бозона Хиггса от одного из свободных параметров НМССМ - tg $\beta$. Сравнивая эти зависимости, легко увидеть, как фаза СР-нарушения изменяет значения ширины распада, в частности, уменьшает это значение. При этом интересно, каким может быть спектр масс остальных бозонов Хиггса. На рис. 4 представлены зависимости массы бозонов Хиггса (в древесном приближении) от фазы СР-нарушения при тех же самых фиксированных значениях свободных параметрах, что и для случаев, представленных на рис. 2 и 3.

НМССМ привлекательна тем, что в её рамках возможно существование достаточно легкого бозона Хиггса, который является ненаблюдаемым на опыте, так как никаких сигналов ниже 125 ГэВ обнаружено не было. В случае нарушения СР-инвариантности это легко объяснить отсутствие у данной частицы определенного СР-четного состояния. Также в НМССМ можно подобрать параметры так, что свойства бозона Хиггса будут отвечать экспериментальным данным. Тогда мы должны предположить, что наблюдаемый нами на LHC бозон является вторым по массе.

\section{ORCID}

Альбина Валентиновна Гурская: http://orcid.org/0000-0003-2543-4932

\section{БИБЛИОГРАФИЧЕСКИЙ СПИСОК}

1. Боос Э. Э. Стандартная модель и предсказания для бозона Хиггса // УФН, 2014. Т. 184, № 9. С. 985-996. doi : 10.3367/UFNr.0184.201409h.0985.

2. Ланев А. В. Результаты коллаборации CMS: бозон Хиггса и поиски новой физики // УФH, 2014. Т. 184, № 9. С. 996-1004. doi: 10.3367/UFNr.0184.201409i. 0996.

3. Казаков Д. И. Хиггсовский бозон открыт: что дальше? // УФН, 2014. Т. 184, № 9. C. 1004-1016. doi: 10.3367/UFNr.0184.201409j.1004.

4. Гурская А. В., Долгополов М. В. Сценарии исследования бозона Хиггса в НМССМ // Вестн. СамГУ. Естественнонаучн. сер., 2014. № 7(118). С. 125-133.

5. Сахаров А. Д. Нарушение СР-инвариантности, С-асимметрия и барионная асимметрия Вселенной // Писъма в ЖКЭТФ, 1967. Т. 5. С. 32-35; Сахаров А. Д. Нарушение СР-инвариантности, С-асимметрия и барионная асимметрия Вселенной // УФН, 1991. T. 161, № 5. C. 61-64. doi : 10.3367/UFNr.0161.199105h.0061.

6. Гурская А. В. СР-нарушение в расширенном секторе Хиггса// Аспирант, 2014. № 4. C. $75-79$. 
7. Ахметзянова Э. Н., Долгополов М. В., Дубинин М. Н. Бозоны Хиггса в двухдублетной модели с нарушением СР-инвариантности // Ядерная физика, 2005. Т. 68, № 11. С. 19131927.

8. Ахметзянова Э. Н., Долгополов М. В., Дубинин М. Н. Суперсимметричная модель с нарушением СР инвариантности. 3. Нарушение СР-инвариантности в хиггсовском секторе// Вестн. СамГУ. Естественнонаучн. сер., 2003. №4(30). С. 147-179, URL: http://vestnik-samgu.samsu.ru/est/2003web4/phys/200340201.pdf (дата обращения: 08.08.2015).

9. Ахметзянова Э. Н., Горбачева И. В., Долгополов М. В., Дубинин М. Н., Смирнов И. А. Суперсимметричная модель с нарушением СР инвариантности. 4. Смешивание в кинетических слагаемых, легкий бозон Хиггса // Вестн. СамГУ. Естественнонаучн. сер., 2004. №2(32). C. 79-109, URL: http://vestnik-samgu.samsu.ru/est/2004web2/phys/ 200420201.pdf (дата обращения: 08.08.2015).

Поступила в редакцию 19/XII/2014;

в окончательном варианте $-27 / \mathrm{V} / 2015$;

принята в печать - 08/VIII/2015.

Vestn. Samar. Gos. Techn. Un-ta. Ser. Fiz.-mat. nauki

[J. Samara State Tech. Univ., Ser. Phys. \& Math. Sci.], 2015, vol. 19, no. 4, pp. 650-657

ISSN: 2310-7081 (online), 1991-8615 (print)

doi: http://dx.doi.org/10.14498/vsgtu1391

MSC: 81T10

THE WIDTH OF THE HIGGS BOSON DECAY INTO TWO PHOTONS IN FRAMEWORK OF NMSSM WITH CP VIOLATION: ONE LOOP APPROXIMATION

\author{
A. V. Gurskaya \\ Samara State University, \\ 1, Academician Pavlov st., Samara, 443011, Russian Federation.
}

\begin{abstract}
The article considers the problem of the extension of standard model physics of elementary particles. We focus on nonminimal supersymmetric models. Nowadays this model is the most appropriate compared with other developed supersymmetric theories. We assume the violation of CP invariance in the Higgs sector. We find a local minimum of the Higgs potential for calculating the physical states of Higgs bosons. This procedure must be performed in order to obtain a stable state of vacuum. Next, calculations of the decay widths of the Higgs boson into two photons there are in this work. We use the method of quantum-field perturbation theory with Feynman diagrams. The results of calculations are presented graphically. We show, that the CP
\end{abstract}

(C) 2015 Samara State Technical University.

Please cite this article in press as:

G u r s k a y A. V. The width of the Higgs boson decay into two photons in framework of NMSSM with CP violation: one loop approximation, Vestn. Samar. Gos. Tekhn. Univ., Ser. Fiz.-Mat. Nauki [J. Samara State Tech. Univ., Ser. Phys. \& Math. Sci.], 2015, vol. 19, no. 4, pp. 650-657. doi: 10.14498/vsgtu1391. (In Russian)

Author Details:

Albina V. Gurskaya (a-gurska@yandex.ru), Postgraduate Student, Dept. of General and Theoretical Physics. 
violating phase affects the value of the decay widths. Also there are dependences of the masses of the Higgs bosons from the phase of CP violation: tree-level approximation. Some model parameters are not determined in the experiment, and concluded in a range of values that gives the possibility to consider various scenarios of multiple Higgs bosons. In particular, several Higgs bosons may be existing with mass less than $125 \mathrm{GeV}$ in case of $\mathrm{CP}$ violation. This can be explained by the absence of a specific CP state of this particle.

Keywords: LHC, Higgs boson, violation of CP invariance, supersymmetry, width of the decay, one-loop correction.

doi: http://dx.doi.org/10.14498/vsgtu1391

\section{ORCID}

Albina V. Gurskaya: http://orcid.org/0000-0003-2543-4932

\section{REFERENCES}

1. Boos E. E. Standard Model and predictions for the Higgs boson, Phys. Usp., 2014, vol. 57, no. 9, pp. 912-923. doi: 10.3367/UFNe.0184.201409h.0985.

2. Lanev A. V. CMS results: Higgs boson and search for new physics, Phys. Usp., 2014, vol. 57, no. 9, pp. 923-930. doi : 10.3367/UFNe.0184.201409i.0996.

3. Kazakov D. I. The Higgs boson is found: what is next?, Phys. Usp., 2014, vol.57, no. 9, pp. 930-942. doi: 10.3367/UFNe.0184.201409j.1004.

4. Gurskaya A. V., Dolgopolov M. V. Scenario study of Higgs boson in the framework NMSSM, Vestnik SamGU. Estestvenno-Nauchnaya Ser., 2014, no.7(118), pp. 125-133 (In Russian).

5. Sakharov A. D. Violation of CP in variance, C asymmetry, and baryon asymmetry of the universe, JETP Lett., 1967, vol. 5, pp. 24-27; Sakharov A. D. Violation of CP in variance, C asymmetry, and baryon asymmetry of the universe, Sov. Phys. Usp., 1991, T. 34, № 5, C. 392-393. doi : 10.1070/PU1991v034n05ABEH002497.

6. Gurskaya A. V. CP violation in the extended Higgs sector, Aspirant, 2014, no. 4, pp. 75-79 (In Russian).

7. Akhmetzyanova É. N., Dolgopolov M. V., Dubinin M. N. Higgs bosons in the two-doublet model involving CP violation, Physics of Atomic Nuclei, 2005, vol. 68, no. 11, pp. 1851-1865. doi: $10.1134 / 1.2131115$.

8. Akhmetzyanova E. N., Dolgopolov M.V., Dubinin M. N. Supersymmetric model with CP violation. 3. CP violation in the Higgs sector, Vestnik SamGU. Estestvenno-Nauchnaya Ser., 2003, no. 4(30), pp. 147-179 (In Russian), Retrieved from http://vestnik-samgu.samsu. ru/est/2003web4/phys/200340201.pdf (August 08, 2015).

9. Akhmetzyanova E. N., Gorbachyeva I. V., Dolgopolov M. V., Dubinin M. N., Smirnov I. A. Supersymmetric model with CP violation. 4. Evidence of the explicit CP violation in the two-Higgs-doublet sector, Vestnik SamGU. Estestvenno-Nauchnaya Ser., 2004, no. 2(32), pp. 79-109 (In Russian), Retrieved from http://vestnik-samgu.samsu.ru/est/2004web2/ phys/200420201.pdf (August 08, 2015).

Received 19/XII/2014;

received in revised form $27 / \mathrm{V} / 2015$;

accepted 08/VIII/2015. 